\title{
Geospatial cellular distribution of cancer-associated fibroblasts significantly impacts clinical outcomes in metastatic clear cell renal cell carcinoma
}

Nicholas H Chakiryan¹, Gregory J Kimmel ${ }^{2}$, Youngchul Kim³ ${ }^{3}$, Joseph Johnson ${ }^{4}$, Noel Clark ${ }^{5}$, Ali Hajiran ${ }^{1}$, Andrew Chang ${ }^{1}$, Ahmet M Aydin ${ }^{1}$, Logan Zemp ${ }^{1}$, Esther Katende ${ }^{1}$, Jad Chahoud ${ }^{1}$, Meghan C Ferrall-Fairbanks ${ }^{2}$, Philippe E Spiess ${ }^{1}$, Natasha Francis ${ }^{1}$, Michelle Fournier ${ }^{1}$, Jasreman Dhillon ${ }^{6}$, Jong Y Park ${ }^{7}$, Liang Wang ${ }^{8}$, James Mulé ${ }^{9}$, Philipp M Altrock ${ }^{2}$, and Brandon J Manley ${ }^{1}$

${ }^{1}$ Department of Genitourinary Oncology, H Lee Moffitt Cancer Center and Research Institute, Tampa, FL, USA

${ }^{2}$ Integrated Mathematical Oncology Department, H Lee Moffitt Cancer Center and Research Institute, Tampa, FL, USA

${ }^{3}$ Department of Biostatistics and Bioinformatics, $\mathrm{H}$ Lee Moffitt Cancer Center and Research Institute, Tampa, FL, USA

${ }^{4}$ Analytic Microcopy Shared Resource, H Lee Moffitt Cancer Center and Research Institute, Tampa, FL, USA

${ }^{5}$ Tissue Core Shared Resource, H Lee Moffitt Cancer Center and Research Institute, Tampa, FL, USA

${ }^{6}$ Department of Pathology, H Lee Moffitt Cancer Center, Tampa, FL, USA;

${ }^{7}$ Department of Cancer Epidemiology, H. Lee Moffitt Cancer Center, Tampa, FL, USA

${ }^{8}$ Department of Tumor Biology, H Lee Moffitt Cancer Center and Research Institute, Tampa, FL, USA

${ }^{9}$ Immunology Department, H Lee Moffitt Cancer Center and Research Institute, Tampa, FL, USA

\section{Corresponding author:}

Nicholas Chakiryan, MD

Department of Genitourinary Oncology, H. Lee Moffitt Cancer Center and Research Institute 12902 Magnolia Drive, Tampa, FL 33612

Tel: 813-745-3208 | Fax: 813-745-8494

Email: Nicholas.chakiryan@moffitt.org

Running title: CAFs cause tumor cell proliferation and poor OS in metastatic ccRCC

\section{Simple Summary}

Cancer-associated fibroblasts are highly prevalent cells in the clear cell renal cell carcinoma (ccRCC) tumor immune microenvironment and are thought to potentiate tumor proliferation primarily through paracrine interactions, as evidenced by laboratory-based studies. We sought to corroborate these findings using surgically removed tissue samples from 96 patients with metastatic cCRCC, and associate geospatial relationships between fibroblasts and rapidly proliferating tumor cells with survival outcomes. We found that cancer-associated fibroblasts exhibited more geospatial clustering with proliferating tumor cells than with dying tumor cells, and that patients whose samples exhibited higher tumor cell proliferation had worse overall survival and were more likely to be resistant to systemic tyrosine-kinase-inhibiting targeted therapies. Immunotherapy resistance was not associated with the geospatial metrics measured in this analysis. Overall, these findings suggest that close proximity to cancer-associated fibroblasts potentiates tumor cell proliferation, worsening survival and conferring resistance to targeted therapies. 


\section{ABSTRACT}

Cancer-associated fibroblasts (CAF) are highly prevalent cells in the tumor microenvironment in clear cell renal cell carcinoma (ccRCC). CAFs exhibit a pro-tumor effect in vitro and have been implicated in tumor cell proliferation, metastasis, and treatment resistance. Our objective is to analyze the geospatial distribution of CAFs with proliferating and apoptotic tumor cells in the ccRCC tumor microenvironment and determine associations with survival and systemic treatment. Pre-treatment primary tumor samples were collected from 96 patients with metastatic ccRCC. Three adjacent slices were obtained from 2 tumor-core regions of interest (ROI) per patient, and immunohistochemistry (IHC) staining was performed for aSMA, Ki-67, and caspase-3 to detect CAFs, proliferating cells, and apoptotic cells, respectively. $\mathrm{H}$-scores and cellular density were generated for each marker. ROls were aligned, and spatial point-patterns were generated, which were then used to perform spatial analyses using a normalized Ripley's $\mathrm{K}$ function at a radius of $25 \mu \mathrm{m}(\mathrm{nK}(25))$. The survival analyses used an optimal cut-point method, maximizing the log-rank statistic, to stratify the IHC-derived metrics into high and low groups, and multivariable Cox regression analyses were performed accounting for age and International Metastatic RCC Database Consortium (IMDC) risk category. Survival outcomes included overall survival (OS) from the date of diagnosis, OS from the date of immunotherapy initiation (OS-IT), and OS from the date of targeted therapy initiation (OS-TT). Therapy resistance was defined as progression-free survival (PFS) $<6$ months, and therapy response was defined as PFS $>9$ months. CAFs exhibited higher cellular clustering with $\mathrm{Ki}-67^{+}$cells than with caspase- $3^{+}$cells (nK(25): Ki-67 1.19; caspase-3 1.05; $P=.04$ ). The median nearest neighbor (NN) distance from CAFs to $\mathrm{Ki}-67^{+}$cells was shorter compared to caspase- $3^{+}$cells $(15$ $\mu \mathrm{m}$ vs $37 \mu \mathrm{m}$, respectively; $P<.001)$. Multivariable Cox regression analyses demonstrated that both high $\mathrm{Ki}-67^{+}$density and $\mathrm{H}$-score were associated with worse OS, OS-IT, and OS-TT. Regarding CAFs, only a high $\mathrm{H}$-score was associated with worse OS, OS-IT, and OS-TT. For caspase $-3^{+}$, high $\mathrm{H}$-score and density were associated with worse OS and OS-TT. Patients whose tumors were resistant to targeted therapy (TT) had higher Ki-67 density and $\mathrm{H}$-scores than those who had TT response. Overall, this ex vivo geospatial analysis of CAF distribution suggests that close proximity clustering of tumor cells and CAFs potentiates tumor cell proliferation, resulting in worse OS and resistance to TT in metastatic CCRCC.

Keywords: metastatic clear cell renal cell carcinoma; cancer associated fibroblasts; Ki-67; spatial analysis; immunohistochemistry

\section{INTRODUCTION}

In normal tissue, fibroblast activation and the subsequent release of cytokines, angiogenic mediators, and growth factors are a physiologic response to tissue injury or stress $[1,2]$. Cancer-associated fibroblasts (CAF) are fibroblasts that have been permanently activated by adjacent tumor cells, which repurpose physiologic fibroblast activity into a pro-tumor survival advantage [1-3]. CAFs are highly prevalent cells in the tumor microenvironment in clear cell renal cell carcinoma (ccRCC) and have been implicated in facilitating tumor cell proliferation, angiogenesis, metastasis, and therapy resistance[4-6]. Several well described pro-tumor properties of CAFs are mediated through hypoxia-inducible-factor-1 (HIF1), a pathway that drives oncogenesis in kidney cancer and is upregulated in the majority of ccRCC tumors via alteration of the von-Hippel Lindau ( $V H L)$ gene [7]. Additionally, molecular receptors mediating the HIF1 pathway are the primary targets for tyrosine-kinase-inhibiting targeted therapies (TT), which are frequently used in metastatic ccRCC [8]. Previous work has demonstrated that increased CAF density in ccRCC tumors is associated with worse overall survival $(\mathrm{OS})[4,6]$. 
Induction of tumor cell proliferation has been postulated as a major mechanism of CAFmediated pro-tumor activity [1]. Immunohistochemical (IHC) staining with Ki-67, a nuclear protein that is present during active phases of the cell cycle and absent from resting cells, has been shown to be an excellent marker for identifying rapidly proliferating tumor cells $[9,10]$. This staining is clinically relevant for grading and prognosis in several primary cancer sites, including breast and colorectal cancers [11-13]. Currently, there is no role for Ki-67 staining in cCRCC guideline-based clinical practice, though a handful of studies have associated high Ki-67 staining with more advanced disease stage, worse OS, and worse cancer-specific survival (CSS) [14-17].

Despite the well described in vitro relationship between CAFs and increased tumor cell proliferation, no prior study has investigated the interplay between CAFs and rapidly proliferating tumor cells in ccRCC tumor samples. Discovery of distinct infiltration patterns and spatial relationships between CAFs and rapidly proliferating tumor cells could support previous in vitro study findings with ex vivo evidence and inform ongoing research into microenvironmentmodulating antineoplastic therapies targeting fibroblasts [18]. Our primary objective was to investigate cellular distribution patterns and spatial relationships between CAFs and proliferating and apoptotic tumor cells in primary tumor samples from patients with metastatic ccRCC and to evaluate the associations between these measures and OS. Our secondary objective was to determine associations between treatment outcomes and targeted therapy and immunotherapy, as well as define spatial relationships between CAFs, proliferating tumors cells, and apoptotic tumor cells.

\section{MATERIALS AND METHODS}

\section{Patient and Sample Selection}

Samples were included from patients with primary ccRCC who had metastatic ccRCC at the time of sample collection and whose tumor specimens were available in formalin-fixed paraffin-embedded blocks. Included patients had received either TT, immunotherapy (IT), or combination TT/IT as systemic treatment from October 2004 to September 2020. Written informed consent was obtained from all tissue donors. All tumor samples were obtained through protocols approved by the institutional review board $(\mathrm{H}$. Lee Moffitt Cancer Center and Research Institute's Total Cancer Care protocol MCC\# 14690; Advarra IRB Pro00014441). Ten specimens of normal kidney tissue were also included as controls. The general workflow of our methods is shown in Figure 1. 


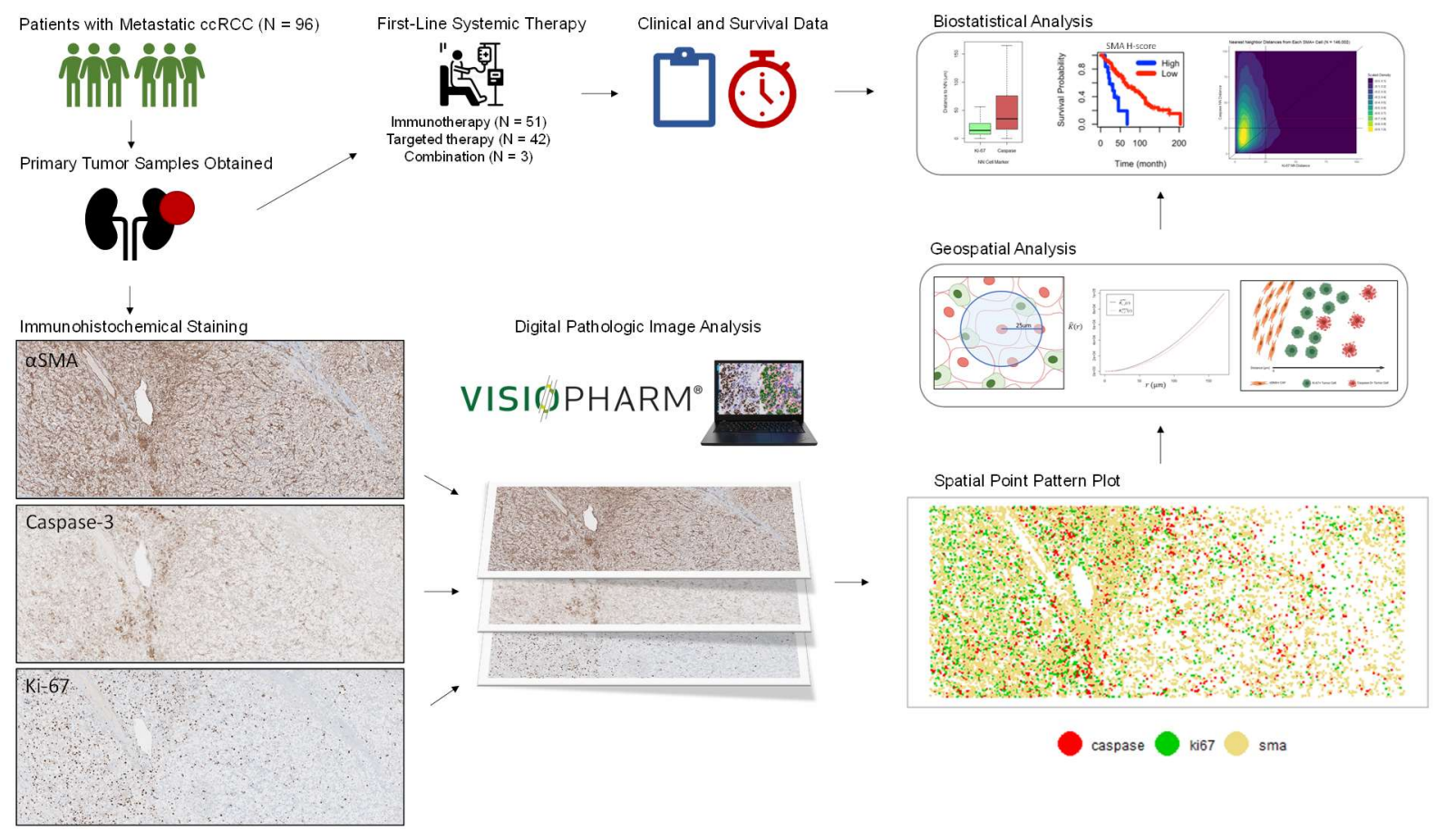

Figure 1: General project workflow for the analysis

\section{Immunohistochemical Specimen Preparation}

Three immediately adjacent slides ( $3 \mu \mathrm{m}$ thickness) were prepared from each tissue block. IHC staining for alpha smooth muscle actin ([aSMA] a marker for activated fibroblasts), Ki-67 (a marker for proliferating cells), and caspase-3 (a marker for cells undergoing apoptosis) were performed; 1 stain was used on each slide. Slides were stained using a Ventana Discovery XT automated system (Ventana Medical Systems, Tucson, AZ) as per manufacturer's protocol, with proprietary reagents. Briefly, slides were deparaffinized on the automated system with EZ Prep solution (Ventana). A heat-induced antigen retrieval method was used in Cell Conditioning (Ventana). The rabbit primary antibody that reacts to Ki-67 (\#790-4286 [Ventana]) was used at a prediluted strength and incubated for 16 minutes. The rabbit primary antibody that reacts to Cleaved Caspase 3 (\#9661 [Cell Signaling, Danvers, MA]) was used at a 1:4000 concentration in Dako antibody diluent (Carpenteria, CA) and incubated for 60 minutes. The rabbit primary antibody that reacts to aSMA, (\#ab32575 [Abcam, Cambridge, MA]) was used at a 1:250 concentration in Dako antibody diluent (Carpenteria, CA) and incubated for 32 minutes. For all stains, the Ventana OmniMap Anti-Rabbit Secondary Antibody was used for 16 minutes. The detection system used was the Ventana ChromoMap kit, and slides were then counterstained with hematoxylin. Slides were then dehydrated and cover-slipped as per normal laboratory protocol. 


\section{Quantitative Digital Image Analysis}

Slides were digitized with a Leica Aperio AT2 slide scanner (Vista, California) using a 20X/0.75NA objective lens. An experienced genitourinary pathologist (JD) used the annotation pen tool in the Aperio Imagescope software to define the tumor-core zone in each hematoxylin and eosin image. High resolution Aperio SVS images representing the $3 \mathrm{IHC}$ stains were imported into Visiopharm (Hoersholm, Denmark) for quantitative digital image analysis. We leveraged a tissue alignment algorithm available in Visiopharm to optimally align 3 adjacent slides of each patient sample set. These slides were visually inspected to ensure appropriate alignment. Two equally sized regions of interest (ROI) were selected from the tumor-core zone from each image; tumor cells were evenly distributed throughout each ROI, and cellular appearance was consistent with that seen in the remainder of the slide. The ROI sizes were standardized at $3426 \times 1379$ pixels, at a pixel resolution of $0.502 \mu \mathrm{m} /$ pixel. Thresholds for staining positivity were set by an experienced digital pathology image analyst (JJ) and confirmed by a study pathologist (JD). These thresholds were used with Visiopharm's cell detection algorithms to identify and categorize cells into negative, weak, moderate, and strong bins on the basis of staining intensity. This intensity-based distribution of cells is comparable to the qualitative method used by pathologists, in which staining intensity is categorized as $0,1+$, $2+$, and $3+[19]$. Percent positivity and $\mathrm{H}$-Scores were calculated for each ROI using this intensity data. $\mathrm{H}$-score is an approach that globally quantifies intensity and percent positivity throughout the entire ROI into 1 score, according to the following formula: $\mathrm{H}$-score $=(1 \times(\%$ cells weak $))+$ $(2 \times(\%$ cells moderate $))+(3 \times(\%$ cells strong $))[20]$. $\mathrm{H}$-scores range from 0 to 300 , with 0 representing no cells staining for the marker of interest and 300 representing every cell staining with the highest intensity[20]. Additionally, Cartesian coordinates for the $(x, y)$ location of each cell's central mass, with each cell's associated marker status, were abstracted from the digital image by using Visiopharm.

\section{Cellular Distribution and Spatial Analysis}

For each ROI, the per-cell Cartesian coordinate and marker positivity data were converted into spatial point patterns. Cell density was calculated as the number of positive cells per $\mathrm{mm}^{2}$. ROls containing $\geq 10$ cells positive for a relevant marker were considered eligible for spatial analysis. As there is no previously validated standard for this cutoff, the $\geq 10$ cell cutoff was agreed upon through consensus of the authors. Cells classified as exhibiting strong staining intensity, as defined above, were considered positive for the purpose of spatial analysis. Cellular clustering was quantified using the Ripley's K function, a methodology for quantifying spatial heterogeneity that is most commonly used in ecology and economics, with isotropic edge correction, and the following normalization was applied: $n K(r)=K(r) / \pi r^{2}$, as described previously[21,22]. As such, the expected value of $\mathrm{nK}(\mathrm{r})$ for complete spatial randomness is 1.0, assuming a homogenous Poisson process[21]. Values of $n K(r)>1.0$ represent cellular clustering and values $<1.0$ represent cellular dispersion. The range of possible values for $n K(r)$ is 0 to infinity. The $\mathrm{nK}(\mathrm{r})$ value is an observed over expected ratio (ie. $\alpha \mathrm{SMA} / \mathrm{Ki}-67 \mathrm{nK}(25 \mu \mathrm{m})=1.30$ can be interpreted as: "There were $30 \%$ more $\mathrm{Ki}-67^{+}$cells within a $25 \mu \mathrm{m}$ radius of each aSMA cell than would be expected if the cells were randomly distributed.").

Initially, 2 search-circle radii were utilized for the spatial analysis. To reflect cellular clustering at a localized distance, $\mathrm{nK}(\mathrm{r})$ at a radius of $25 \mu \mathrm{m}$ was used in this analysis and will 
henceforth be referred to as $\mathrm{nK}(25)$. The search-circle radius value of $25 \mu \mathrm{m}$ was selected, as it represents approximately double that of a typical ccRCC tumor cell radius and as such should represent the area in the immediate vicinity of the cell. To reflect a more global view of cellular clustering, a radius of $125 \mu \mathrm{m}$ was utilized, which will be referenced as $\mathrm{nK}(125)$. Subsequently, we determined that Spearman's correlations demonstrated a very strong correlation between $n K(25)$ and $n K(125)(r>0.90)$. Given this redundancy, we utilized $n K(25)$, not $n K(125)$, for the remainder of the analysis.

As a second independent measure of spatial analysis, linear nearest neighbor distances were determined from each cell to its nearest neighbor cell among each of the 3 cell types. Scaled two-dimensional kernel density plots were generated to analyze the distribution of nearest neighbor distances from each aSMA ${ }^{+}$cell to its nearest neighbor caspase- $3^{+}$and $\mathrm{Ki}-67^{+}$ cell. These nearest neighbor density plots were generated with the cohort stratified by ccRCC versus normal kidney, treatment response versus resistance to TT, and treatment response versus resistance to IT.

\section{Variable Definitions}

Age was defined as life years at the time of $\mathrm{cCRCC}$ diagnosis. Tumor grade was defined per the histologic classification criteria proposed by the International Society of Urologic Pathologists and implemented by the World Health Organization (ISUP/WHO)[23]. International Metastatic RCC Database (IMDC) scores were determined for each patient and categorized into good, intermediate, and poor risk groups, as previously described[24]. OS was determined from the date of metastatic CCRCC diagnosis to the date of death or censoring at last follow-up. Treatments classified as IT included both immune checkpoint inhibitors and high-dose interleukin-2 (IL-2). Treatments classified as TT included small-molecule tyrosine-kinaseinhibiting therapies approved for first-line treatment in cCRCC[17]. OS from the date of first receipt of IT (OS-IT) and OS from the date of first receipt of TT (OS-TT) were also determined. Response to therapy was defined as clinical progression-free survival greater than 9 months from treatment initiation, and resistance was defined as progression $<6$ months from treatment initiation. Patients who progressed between 6 and 9 months after initiating therapy were not classified as responsive or resistant. Statistical significance was defined as a two-tailed alpharisk of 0.05 or less.

\section{Statistical Analysis}

In addition to demographic, clinical, and pathologic characteristics, patient-level data included marker densities and intensities, $\mathrm{H}$-scores, and uni- and bi-variate spatial distribution metrics for each of the 3 included IHC markers. As each patient had 2 ROls analyzed, the IHCderived metrics were averaged such that each patient had 1 value for each metric. Spearman's correlation coefficients were determined between each pairwise combination to assess the interactions between the $\mathrm{IHC}$-derived metrics.

As standardized cutoffs do not exist for the IHC-derived metrics utilized in this analysis, optimal cut-points were determined for each metric, maximizing the log-rank test statistics for OS, as previously described[25]. The survival analysis used a multivariable Cox proportional hazards regression for each metric, utilizing age and IMDC risk category as covariates. False discovery rate- (FDR-) adjusted $P$ values were calculated for multiple comparison correction. This analysis was repeated for the OS-IT and OS-TT endpoints. 
Patients were then stratified by response or non-response to IT and TT, and values of each IHC-derived metric were compared between responders and non-responders using a twosample t-test.

The distribution of nearest neighbor distances from aSMA ${ }^{+}$cells to their nearest caspase $-3^{+}$cell was compared to that of the distance to the nearest Ki-67-cell using a Wilcoxon test. This process was repeated after stratifying the cohort by IT response and non-response and TT response and non-response.

\section{Statistical Software}

All statistical and spatial analyses were performed using $R$ version 4.0.2 (The $R$ Foundation for Statistical Computing [Vienna, Austria]). The maxstat package was used to determine optimal cut-points, survival and survminer packages used for the survival analysis, and the spatstat, MASS, and FNN packages for spatial analysis.

\section{RESULTS}

\section{Study Population}

The study population included 96 patients (median age, 60 years (IQR 33-87); male, 68 (71\%); White race, 90 [94\%]) (Table 1). Fifty-one patients (53\%) underwent first-line IT (18 responders, 25 resistant, 8 indeterminate), 42 patients (44\%) had first-line TT (27 responders, 9 resistant, 6 indeterminate), and 3 patients (3\%) had first-line combination IT/TT. Median followup was 54 months (IQR, 41-97) for the 37 patients (38\%) who were alive at last contact.

Table 1. Baseline patient, tumor, and treatment characteristics, $\mathbf{N}=96^{1}$

\begin{tabular}{cc}
\hline Characteristic & No. (\%) \\
Median age, range & $59(55-67)$ \\
Gender & \\
Female & $28(29)$ \\
Male & $68(71)$ \\
Race & \\
White & $90(94)$ \\
Black & $2(2.1)$ \\
Asian & $1(1.0)$ \\
Other & $3(3.1)$ \\
IMDC Risk Category & $20(21)$ \\
Favorable & $56(58)$ \\
Intermediate & $9(9)$ \\
Poor & $11(12)$ \\
Indeterminate & $8.2(6.0,11.0)$ \\
Primary tumor size (cm) & $11(11)$ \\
ISUP Grade & $60(62)$ \\
2 & $25(26)$ \\
3 & $87(91)$ \\
4 & $9(9.4)$
\end{tabular}




$\begin{array}{cc}\text { Rhabdoid Variant } & \\ \text { No } & 88(92) \\ \text { Yes } & 8(8.3) \\ \text { First Line Therapy } & 51(53) \\ \text { IT } & 42(44) \\ \text { TT } & 3(3.1) \\ \text { Both } & 25(32) \\ \text { Response Category } & 18(23) \\ \text { IT Resistance } & 8(8.3) \\ \text { IT Response } & 9(9.4) \\ \text { IT Indeterminate } & 27(28) \\ \text { TT Resistance } & 6(6.3) \\ \text { TT Response } & \\ \text { TT Indeterminate } & \\ \text { 1 Statistics presented: median (IQR) } \\ \text { Pational Metastatic RCC Database Consortium; ISUP, International Society of Urologic } \\ \text { Pathologists; IT, immunotherapy; TT, targeted therapy }\end{array}$

CAFs are highly prevalent in the ccRCC tumor microenvironment

$\alpha \mathrm{SMA}^{+} \mathrm{CAFs}$ had the highest median cell density and $\mathrm{H}$-score (density, $1904 \mathrm{cells} / \mathrm{mm}^{2}$ [IQR 1266-3382]; $\mathrm{H}$-score 35.5 [IQR 24.0-51.2]), as compared with respective values for Ki-67 ${ }^{+}$ and caspase-3 $3^{+}$tumor cells (Ki-67 ${ }^{+}$density, 1087 cells/mm ${ }^{2}$ [IQR, 433-1909]; Ki-67 H-score 7.0 [IQR 3.1-15.5]; caspase-3 ${ }^{+}$density, 203 cells $/ \mathrm{mm}^{2}$ [IQR $\left.64-547\right]$; caspase-3 ${ }^{+} \mathrm{H}$-score, 0.8 [IQR, 0.3-2.2]) (Wilcoxon $P<.001$ for all comparisons within density and $\mathrm{H}$-score groups)(Figure 2a).

\section{CAFs are spatially clustered with proliferating tumor cells}

Using the normalized Ripley's $\mathrm{K}$ function, we found that $\alpha \mathrm{SMA}^{+} \mathrm{CAFs}$ were more spatially clustered with $\mathrm{Ki}-67^{+}$tumor cells than they were with caspase- $3^{+}$tumor cells $(\mathrm{nK}(25)$, 1.15 vs 1.09 , respectively; $P=.045$ )(Figure 2 b). Caspase- $3^{+}$tumor cells were more spatially clustered with $\mathrm{Ki}-67^{+}$tumor cells than they were with $\alpha \mathrm{SMA}^{+} \mathrm{CAFs}$ (nK(25), 1.16 vs 1.09 , respectively; $P=.035)$. The amount of cellular clustering demonstrated between $\alpha \mathrm{SMA}^{+} \mathrm{CAFs}$ and $\mathrm{Ki}-67^{+}$cells was similar to that between $\mathrm{Ki}-67^{+}$tumor cells and caspase- $3^{+}$tumor cells $(P=$ $.434)$.

The median nearest-neighbor (NN) distance from $\alpha \mathrm{SMA}^{+} \mathrm{CAFs}$ to the nearest $\mathrm{Ki}-67^{+}$cell was shorter than the distance to the nearest caspase- $3^{+}$cell $(14.4$ vs $34.9 \mu \mathrm{m} ; P<.001)$ (Figure 2c). The median $\mathrm{NN}$ distance from $\mathrm{Ki}-67^{+}$cells to the nearest caspase- $3^{+}$cell was shorter than the distance from aSMA ${ }^{+}$CAFs to the nearest caspase- $3^{+}$cell $(31.3$ vs $34.9 \mu \mathrm{m} ; P<.001)$. 
A

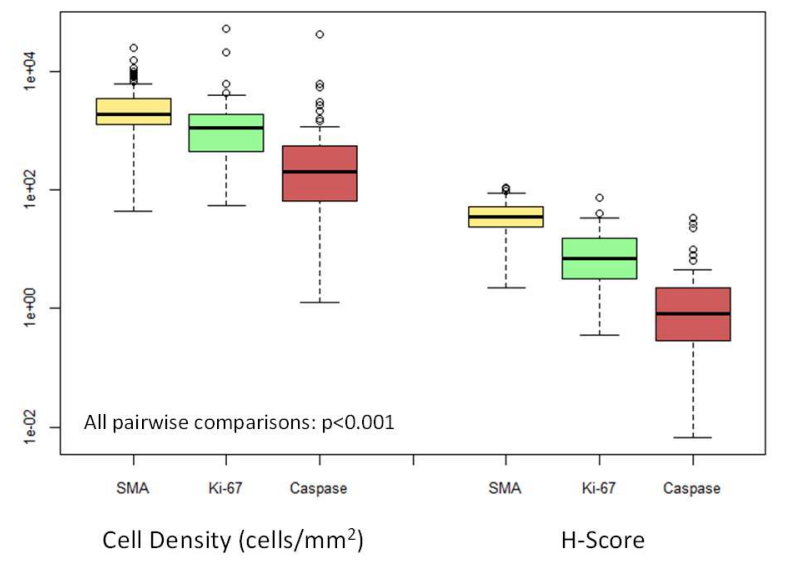

B

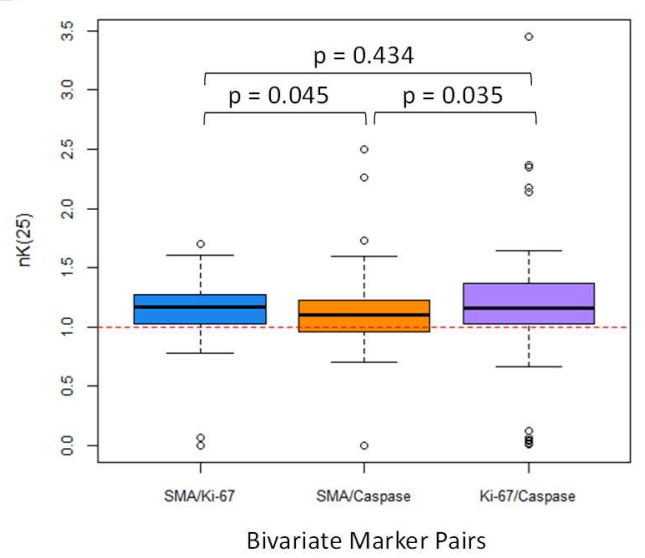

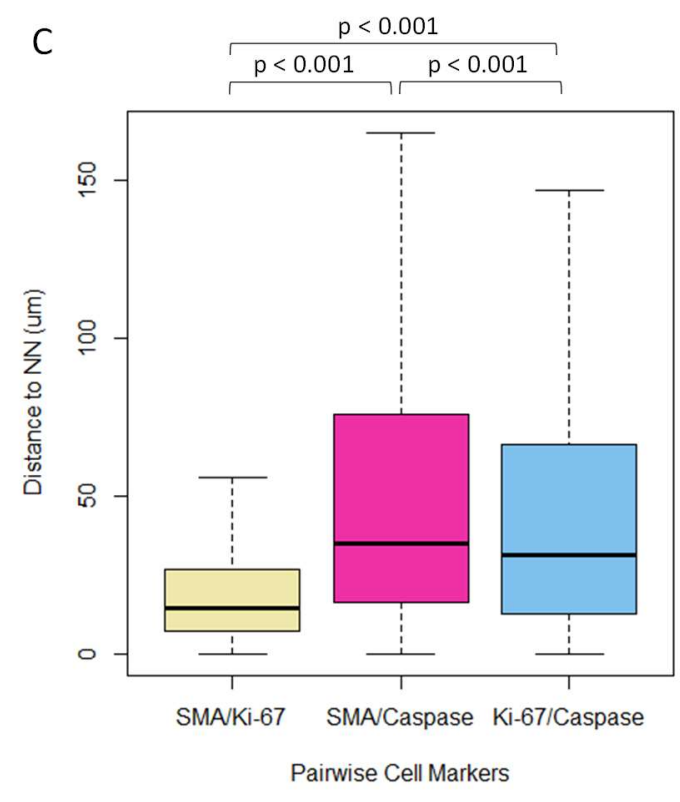

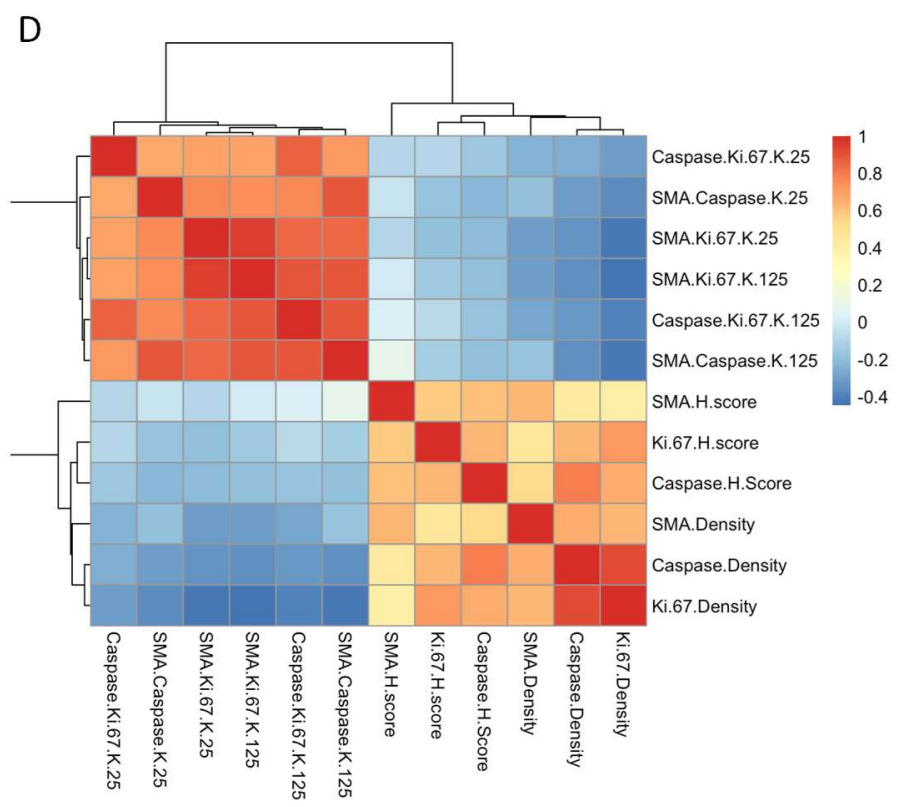

Figure 2: A - Boxplot diagrams depicting median cell density (cells $/ \mathrm{mm}^{2}$ ) and $\mathrm{H}$-scores for each of the immunohistochemistry stains used in the analysis (aSMA, Ki-67, and caspase-3).

Wilcoxon $P$ values $<.001$ for all comparisons. B - Boxplot diagrams depicting bivariate spatial clustering, $\mathrm{nK}(25)$ and values for each pairwise combination of cells for patient-level data $(\mathrm{n}=$ 96 patients). Values $>1.0$ reflect clustering of the paired cells, and values $<1.0$ reflect dispersion. Wilcoxon $P$ values displayed in the plot. $C$ - Boxplots depicting median nearest neighbor distances in $\mu \mathrm{m}$ from the indicated pairwise cell combinations for cellular-level data $(\mathrm{n}$ $>500000$ cells). Wilcoxon $P$ values displayed in the plot. D - Heatmap diagram depicting Spearman's correlation coefficients between each immunohistochemistry-derived measurement used in the analysis. Note that the deepest blue values reflect a Spearman's correlation coefficient of -0.4 and the deepest red 1.0 .

Overall, the density and spatial analyses suggest a tumor architecture in which aSMA ${ }^{+}$ CAFs and $\mathrm{Ki}-67^{+}$tumor cells demonstrate the highest clustering and shortest NN distance, 
$\mathrm{aSMA}^{+} \mathrm{CAFs}$ and caspase- $3^{+}$tumor cells have the least amount of clustering and longest NN distance, and cell densities are highest for $\alpha \mathrm{SMA}^{+} \mathrm{CAFs}$ and lowest for caspase- $3^{+}$tumor cells. Figure 3 shows an example of the hypothetical median tumor architecture of these 3 markers, as suggested by the distribution and spatial analysis metrics for the entire cohort.
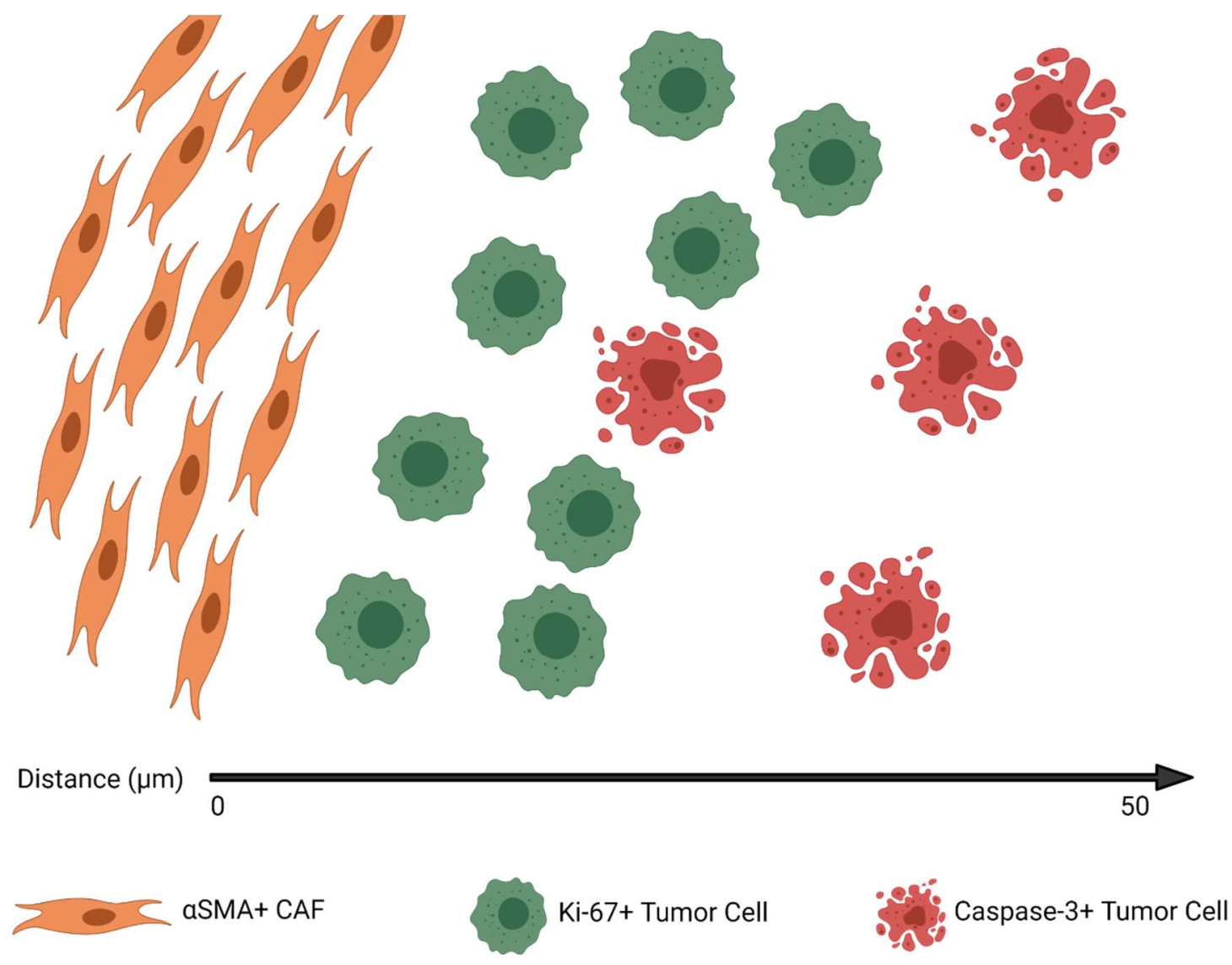

Figure 3: Median tumor architecture identified on immunohistochemistry, derived from the marked cell density and clustering metrics described in Figure 2.

CAF density and aSMA H-score are positively correlated with proliferating and apoptotic tumor cells

Spearman's correlations demonstrated that the aSMA H-score had a moderate positive correlation ( $r=0.4-0.6)$ with aSMA density, Ki-67 H-score, and Caspase $\mathrm{H}$-score and a weak positive correlation ( $r=0.2-0.4$ ) with caspase-3 density and Ki-67 density (Figure 2d). $\alpha S M A$ density had a moderate positive correlation with Ki-67 density, caspase-3 density, and aSMA Hsore and a weak positive correlation with $\mathrm{Ki}-67 \mathrm{H}$-score and capsase- $3 \mathrm{H}$-score. Clustering metrics and density/ $\mathrm{H}$-score metrics were consistently found to have either no correlation or a weak negative correlation $(r=-0.4-0)$. Clustering metrics at a local or global scale $(n K(25)$ or $\mathrm{nK}(125)$, respectively) were found to have a strong positive correlation with each other, within marker-types $(r=0.6-1.0)$. Complete information regarding pairwise correlations between the IHC-derived metrics can be found in Figure 2d. 
None of the IHC-derived metrices were significantly correlated with primary tumor size or grade, confirming that these measures are not simply surrogates for conventionally measures (Supplemental Figure 1).

High aSMA, Ki-67, and caspase-3 H-scores are associated with significantly worse OS

Multivariable Cox proportional hazards regression analysis showed that patients with a high aSMA H-score had significantly worse OS (HR 1.45 [1.05-2.01]; $P=.02)$, OS-IT (HR 1.51 [1.05-2.17]; $P=.03$ ), and OS-TT (HR 1.46 [1.06-2.02]; $P=.012$ than patients with a low aSMA $\mathrm{H}$-score. Interestingly, stratification by aSMA CAF cell density alone had no statistically significant association with OS, OS-IT, or OS-TT $(P=.32,0.17$, and 0.24 , respectively) compared with patients with low $\alpha \mathrm{SMA}^{+} \mathrm{CAF}$ cell density (Figure 4a).

Patients with a high Ki-67 H-score had significantly worse OS (HR 1.79 [1.40-2.29]; $P<$ $.001)$, OS-IT (HR 1.47 [1.13-1.91]; $P<.001)$, and OS-TT (HR 1.71 [1.35-2.17]; $P<.001)$ than patients with a low Ki-67 $\mathrm{H}$-score. Patients with high $\mathrm{Ki}-67^{+}$cell density had significantly worse OS (HR, 1.30 [1.06-1.61]; $P=.01)$, and OS-TT (HR, 1.27 [1.04-1.57]; $P=.02)$, with no significant difference in OS-IT (HR, 1.21 [0.98-1.50]; $P=.08)$, than patients with low $\mathrm{Ki}-67^{+}$cell density (Figure 4b).

Patients with a high caspase-3 H-score had significantly worse OS (HR 1.33 [1.01-1.75]; $P=.04$ ) and OS-TT (HR 1.46 [1.14-1.88]; $P<.001$ ), with no significant difference in OS-IT (HR 1.19 [0.89-1.58]; $P=.24)$, than patients with low caspase- $3 \mathrm{H}$-scores. Patients with high caspase- $3^{+}$cell density had significantly worse OS (HR 1.26 [1.02-1.56]; $P=.03$ ), and OS-TT (HR 1.25 [1.02-1.53]; $P=.03$ ), with no significant difference in OS-IT (HR 1.18 [0.95-1.46]; $P=$ .13) (Figure 4c). 


\section{OS}

A

SMA.H.score Strata + High + Low

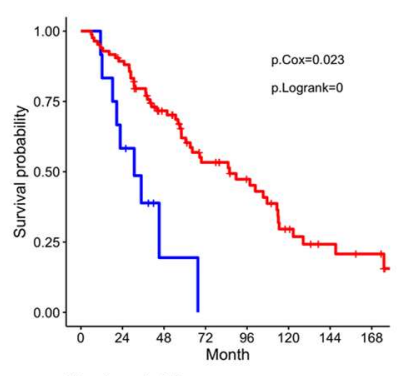

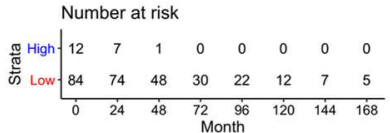

B

Ki.67.H.score Strata $-\mathrm{High}+$ Low

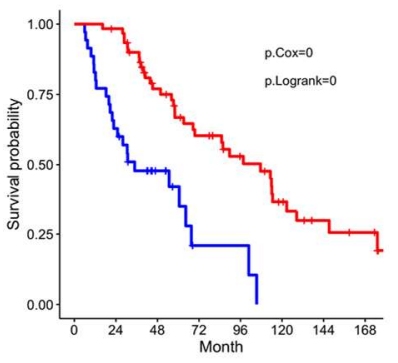

Number at risk

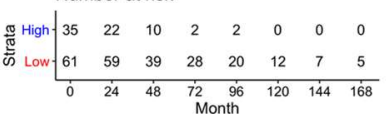

C

Caspase.H.Score

Strata + High + Low

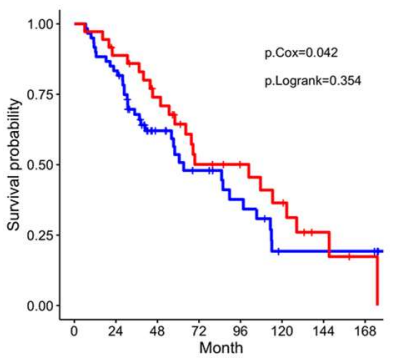

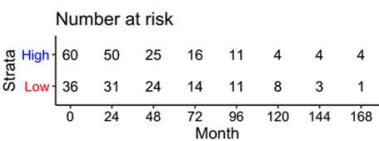

OS-IT

SMA.H.score

Strata + High + Low

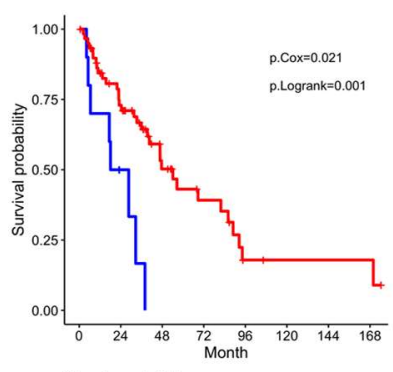

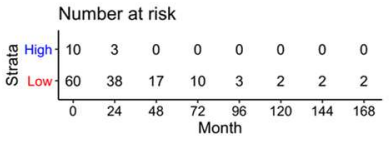

Ki.67.H.score

Strata - High - Low

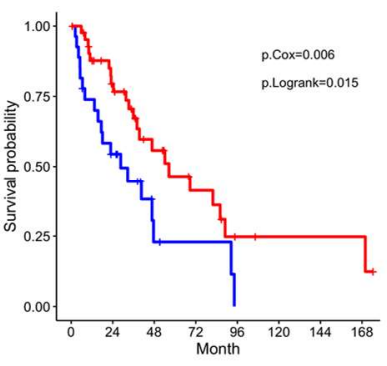

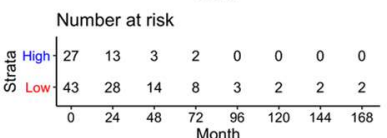

Caspase.H.Score

Strata + High + Low

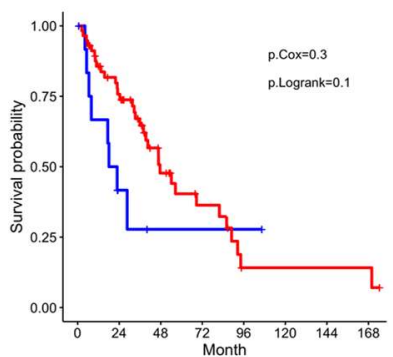

Number at risk

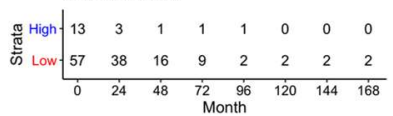

OS-TT

SMA.H.score Strate + High + Low

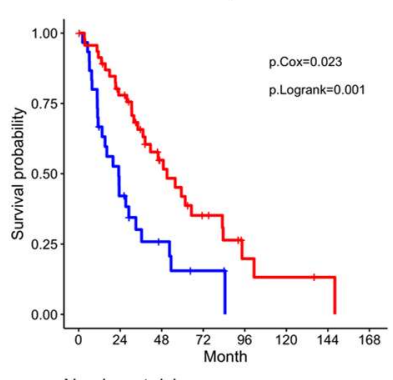

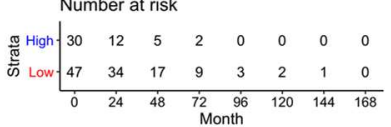

Ki.67.H.score
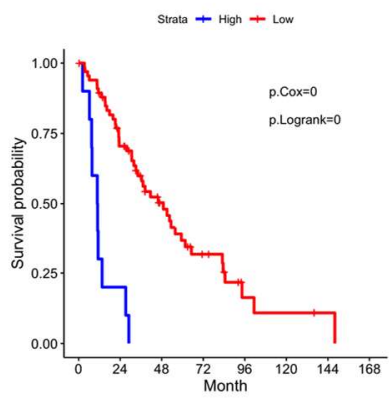

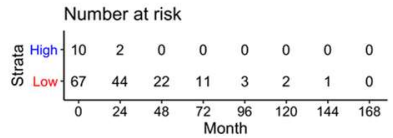

Caspase.H.Score

Strata + High + Low

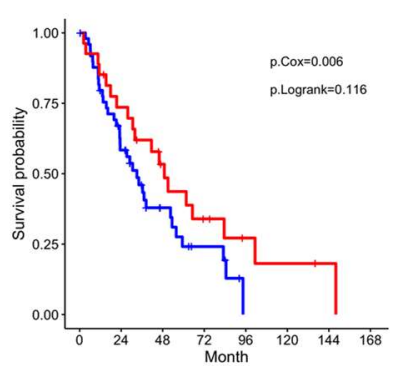

Number at risk

क Low $\begin{array}{ccccccccc}50 & 27 & 11 & 5 & 0 & 0 & 0 & 0 \\ 27 & 19 & 11 & 6 & 3 & 2 & 1 & 0 \\ 0 & 24 & 48 & 72 & 96 & 120 & 144 & 168\end{array}$

Figure 4: Kaplan-Meier curves for overall survival, overall survival for the date of immunotherapy initiation, and overall survival from the date of targeted therapy initiation, with groups stratified 
by $\mathrm{A}$ : aSMA $\mathrm{H}$-score, $\mathrm{B}$ : Ki-67 $\mathrm{H}$-score, and $\mathrm{C}$ : Caspase $\mathrm{H}$-score. Multivariable Cox regression and log-rank $P$ values reported within the plots.

Using the Ripley's K function, we did not find any spatial clustering metrics predictive of OS, OS-IT, or OS-TT. The results of the multivariable Cox regression analysis assessing all $\mathrm{IHC}$-derived $\mathrm{H}$-scores, densities, and spatial clustering metrics are available in Table 2.

Table 2. Multivariable Cox regressions using the covariates age and International Metastatic RCC Database Consortium risk category for the outcomes of A. overall survival, B. overall survival from the date of immunotherapy initiation, C. overall survival from the date of targeted therapy initiation.

A

\begin{tabular}{|c|c|c|c|c|c|c|c|c|}
\hline Metric & Cutoff & N High & $\mathrm{N}$ Low & Survival High (mo.) & Survival Low (mo.) & $\begin{array}{l}\text { Cox } \\
\text { HR }\end{array}$ & $95 \% \mathrm{Cl}$ & $P$ \\
\hline SMA H score & 37.028 & 12 & 84 & 63.2 & 97.6 & 1.45 & $\begin{array}{l}1.05- \\
2.01\end{array}$ & .02 \\
\hline Ki $67 \mathrm{H}$ score & 7.088 & 35 & 61 & 54.7 & 97.6 & 1.79 & $\begin{array}{l}1.40- \\
2.29\end{array}$ & $<.001$ \\
\hline Caspase H Score & 0.834 & 60 & 36 & 85.8 & 68.6 & 1.33 & $\begin{array}{l}1.01- \\
1.75\end{array}$ & .04 \\
\hline SMA Density & 1892.363 & 48 & 48 & 85.1 & 63.2 & 1.17 & $\begin{array}{c}0.86- \\
1.60\end{array}$ & .32 \\
\hline Ki 67 Density & 1097.034 & 48 & 48 & 64.4 & 85.1 & 1.30 & $\begin{array}{l}1.06- \\
1.61\end{array}$ & .01 \\
\hline Caspase Density & 205.198 & 47 & 49 & 85.8 & 68.6 & 1.26 & $\begin{array}{c}1.02- \\
1.56\end{array}$ & .03 \\
\hline SMA-Ki67 nK(25) & 1.167 & 46 & 48 & 69.6 & 68.6 & 0.98 & $\begin{array}{c}0.71- \\
1.34\end{array}$ & .90 \\
\hline SMA-Caspase nK(25) & 1.106 & 38 & 44 & 85.1 & 67.6 & 0.92 & $\begin{array}{c}0.65- \\
1.30\end{array}$ & .64 \\
\hline Caspase-Ki67 nK(25) & 1.163 & 42 & 42 & 67.6 & 89.7 & 0.82 & $\begin{array}{c}0.58- \\
1.16\end{array}$ & .26 \\
\hline
\end{tabular}

B

\begin{tabular}{|c|c|c|c|c|c|c|c|c|}
\hline Metric & Cutoff & N High & N Low & Survival High (mo.) & Survival Low (mo.) & $\begin{array}{l}\text { Cox } \\
\text { HR }\end{array}$ & $95 \% \mathrm{Cl}$ & $P$ \\
\hline SMA H score & 36.346 & 10 & 60 & 32.6 & 47.6 & 1.51 & $\begin{array}{l}1.05- \\
2.17\end{array}$ & .03 \\
\hline Ki 67 H score & 7.06 & 27 & 43 & 33.3 & 56.5 & 1.47 & $\begin{array}{l}1.13- \\
1.91\end{array}$ & $<.001$ \\
\hline Caspase H Score & 1.137 & 13 & 57 & 46.7 & 46.7 & 1.19 & $\begin{array}{l}0.89- \\
1.58\end{array}$ & .24 \\
\hline SMA Density & 1911.912 & 35 & 35 & 46.7 & 35.9 & 1.24 & $\begin{array}{l}0.91- \\
1.68\end{array}$ & .17 \\
\hline Ki 67 Density & 1097.034 & 36 & 34 & 40.5 & 46.7 & 1.21 & $\begin{array}{l}0.98- \\
1.50\end{array}$ & .08 \\
\hline Caspase Density & 189.285 & 35 & 35 & 86.1 & 40.5 & 1.18 & $\begin{array}{l}0.95- \\
1.46\end{array}$ & .13 \\
\hline SMA-Ki67 nK(25) & 1.139 & 35 & 33 & 40.5 & 46.7 & 0.94 & $0.67-$ & .70 \\
\hline
\end{tabular}




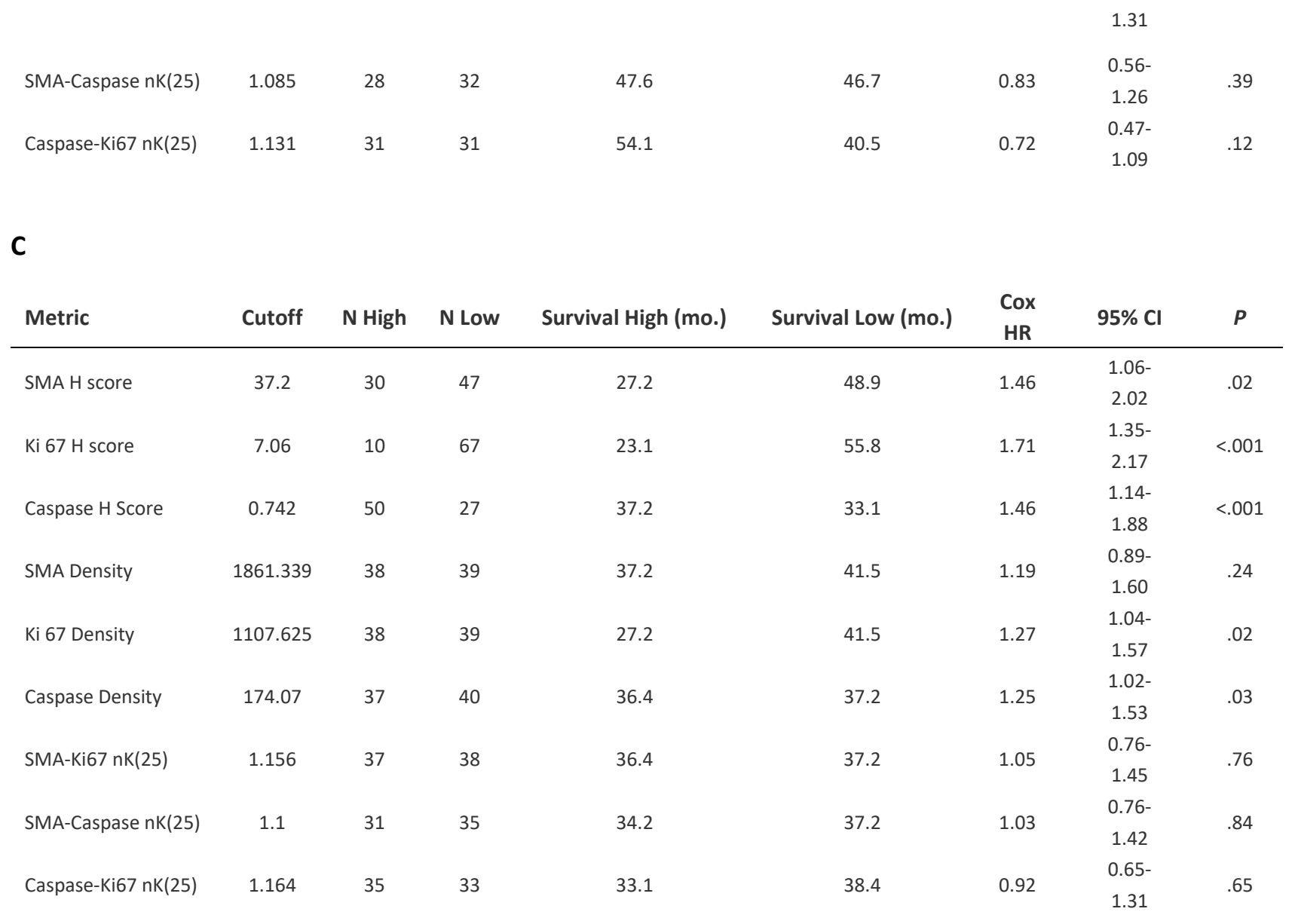

High Ki-67 density and $\mathrm{H}$-score are associated with resistance to first-line TT

To examine specific outcomes to first-line therapy, we stratified patients by response and resistance to TT or IT. Patients who were resistant to TT had higher Ki-67 H-scores and higher $\mathrm{Ki}-67^{+}$cell densities (Wilcoxon $P$ values $=.013$ and .035 , respectively; Figure 5). None of the other IHC-derived metrics were associated with TT or IT response or resistance in the first line setting. 
A

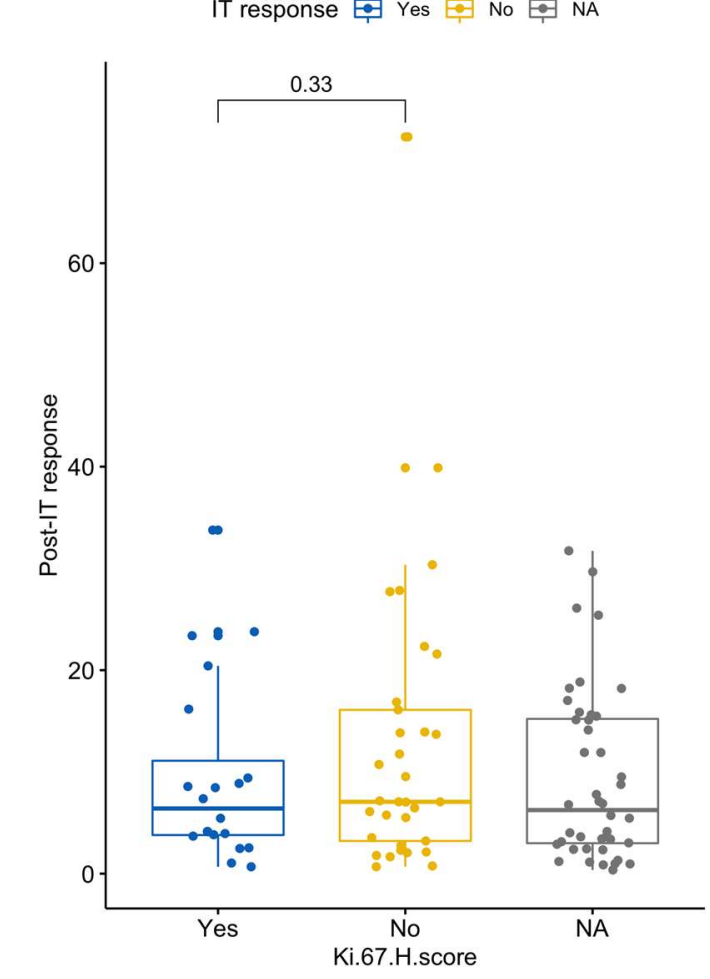

B

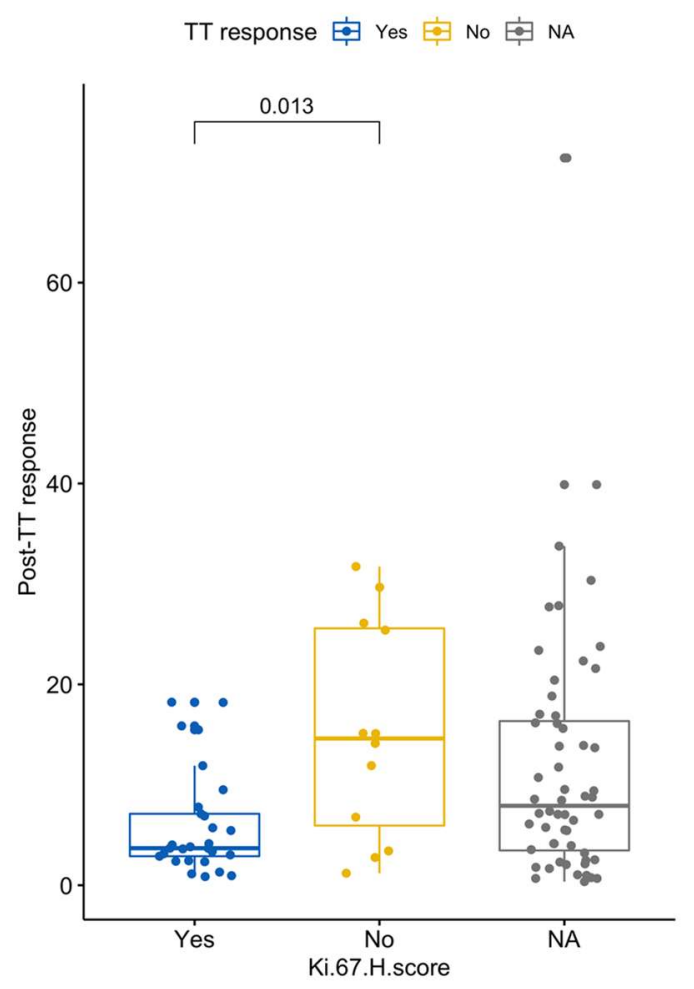

IT response 审 Yes 亩 No 审 NA

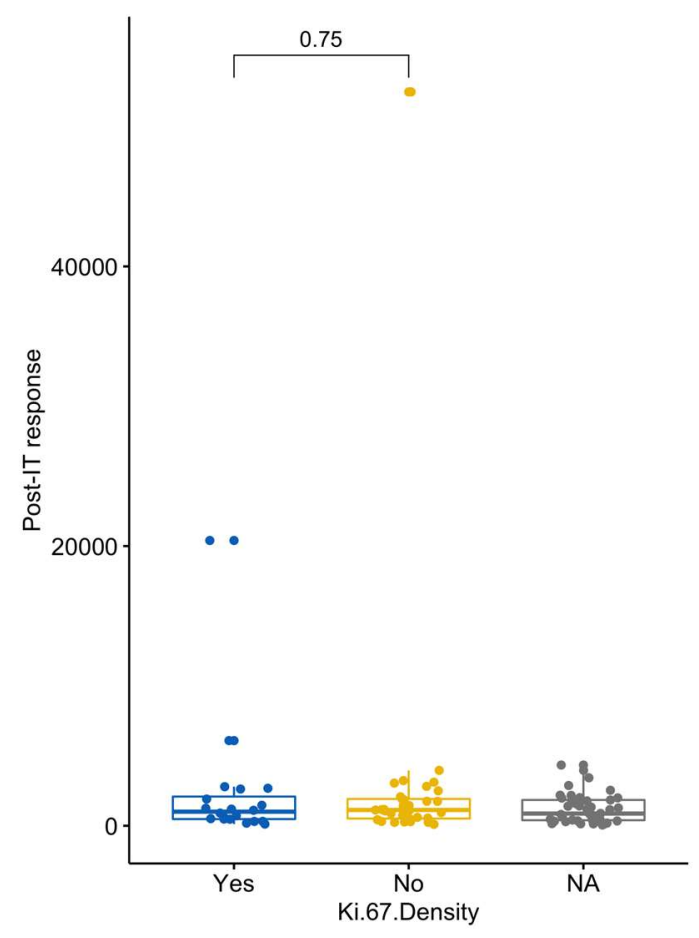

TT response 审 Yes

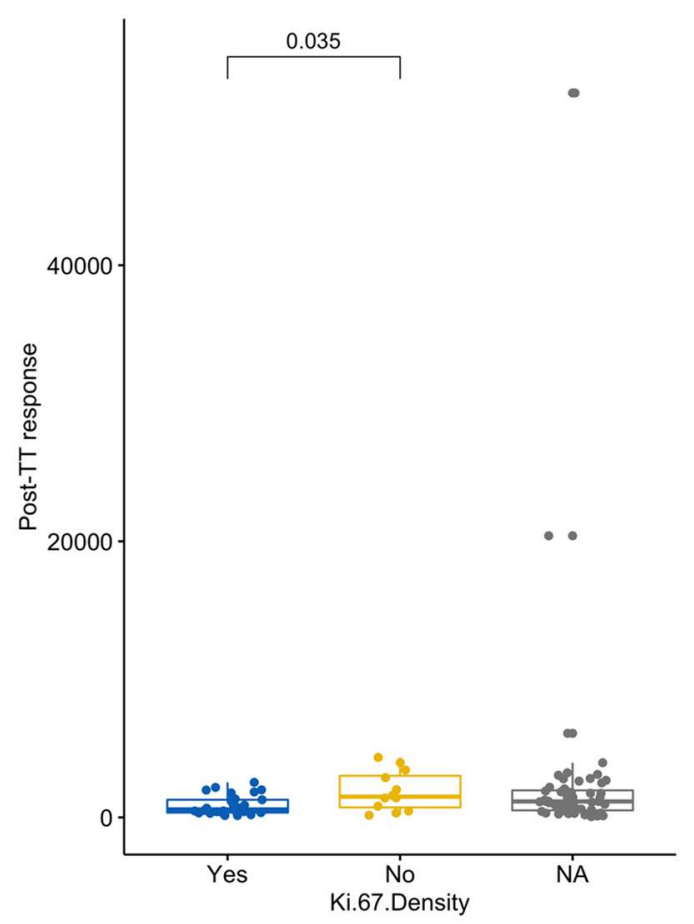

Figure 5: Ki-67 $\mathrm{H}$ scores and densities stratified by response to first-line therapy with $\mathrm{A}$ : 
Immunotherapy and B: Targeted therapy. The NA category represents patients that did not receive that respective therapy as a first-line agent.

Distinct geospatial distributions of CAFs are associated with treatment response

Examining our cellular marker distributions, we found ccRCC and normal kidney samples to have dramatically different kernel density distributions with respect to their NN distances from each marked cell type (Figure 5A.i and 5A.ii). ccRCC samples had a shorter NN distance from $\alpha \mathrm{SMA}^{+}$to $\mathrm{Ki}-67^{+}$cells, compared with normal kidney samples (13 vs $31 \mu \mathrm{m}$, respectively; $P<.001$ ), and longer NN distance from aSMA ${ }^{+}$to caspase $-3^{+}$cells (32 vs $9 \mu \mathrm{m}$, respectively; $P<.001$ ) (Figure 6.A.iii).

TT-responsive and TT-resistant patient samples had significantly different kernel density distributions with respect to their NN distances from each marked cell type (Figure 6B.i and 5B.ii). TT-responsive patient samples had a longer NN distance from $\alpha \mathrm{SMA}^{+}$to caspase- $3^{+}$ cells (31 vs $21 \mu \mathrm{m}$, respectively; $P<.001$ ) (Figure 6.B.iii).

IT-responsive and IT-resistant patient samples had significantly different kernel density distributions with respect to their NN distances from each marked cell type (Figure 6C.i and 6C.ii). IT-responsive patient samples had shorter NN distance from $\alpha \mathrm{SMA}^{+}$to caspase- $3^{+}$cells (24 vs $35 \mu \mathrm{m}$, respectively; $P<.001$ ) (Figure 6.C.iii). 
A
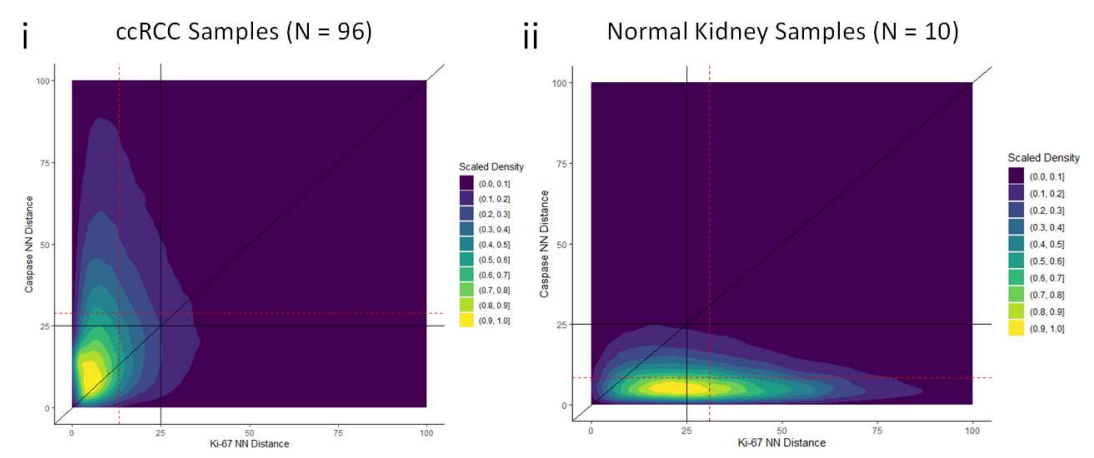

iii

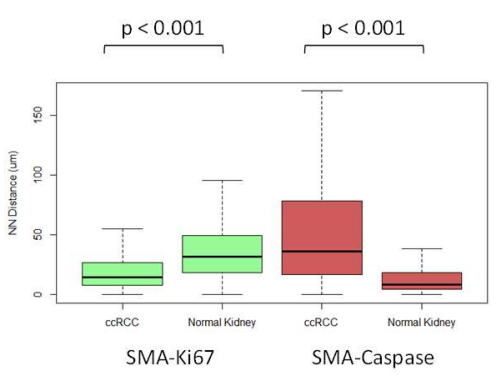

B

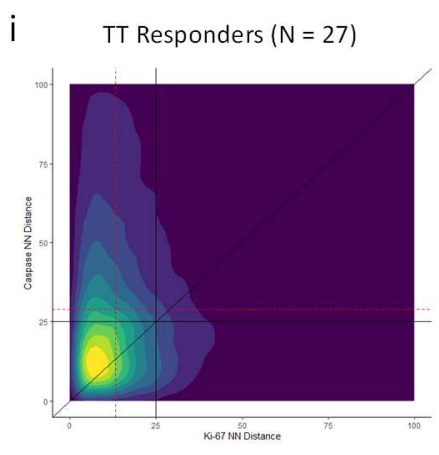

ii

TT Resistant ( $\mathrm{N}=9$ )
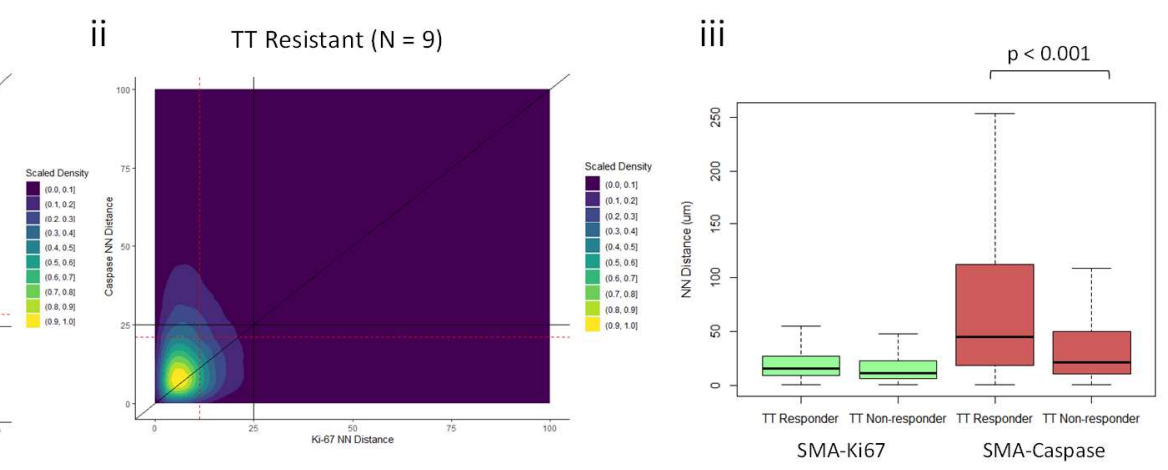

C

IT Responders ( $N=18$ )

ii

IT Resistant ( $N=25)$

iii
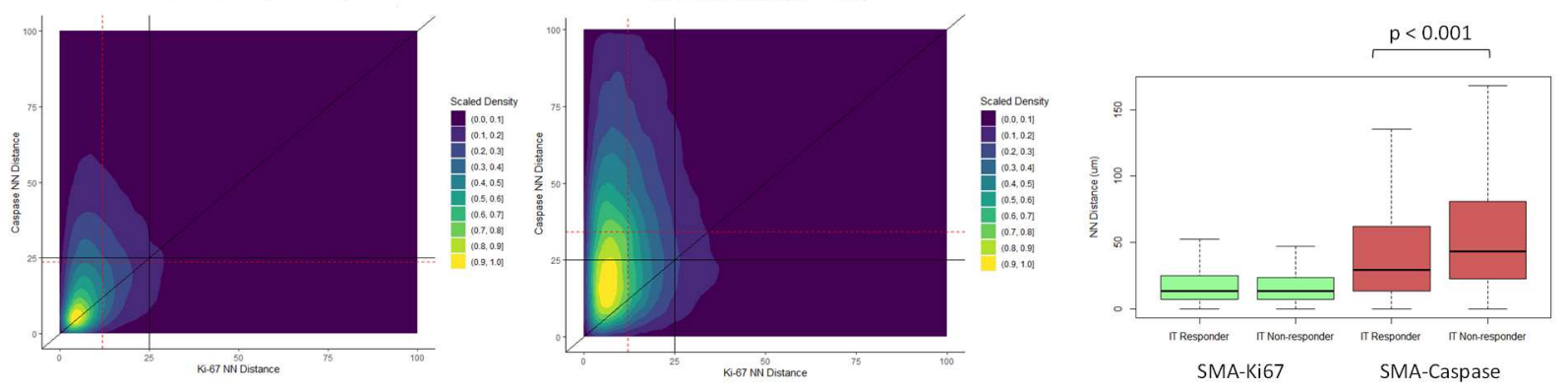

Figure 6: Scaled kernel density plots (i and ii's), with the x-axis representing the distance from each $\alpha \mathrm{SMA}^{+}$cell to its nearest $\mathrm{Ki}-67^{+}$neighbor $(\mu \mathrm{m})$ and the $y$-axis representing the distance from each $\alpha \mathrm{SMA}^{+}$cell to its nearest capsase- $3^{+}$neighbor $(\mu \mathrm{m})$. Each point on the plot represents an individual $\alpha \mathrm{SMA}^{+}$cell, and the location of the point corresponds with cells' NN distance from the nearest $\mathrm{Ki}-67^{+}$and caspase $-3^{+}$neighbor. Two-dimensional kernel density is graphically depicted, overlying the scatter plot, and has been scaled to a range of 0-1.0 in each plot. Dotted red lines reflect median values for the $x$ and $y$ axes. Solid black lines are anchored at fixed values to facilitate visual interpretation between plots. Boxplots (iii's) compare median

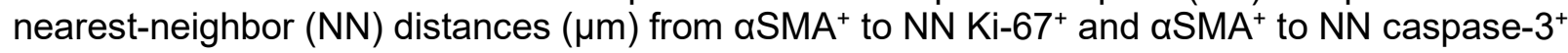
cells, stratified by groups as indicated. A.i - All ccRCC samples $(N=96)$, A.ii - Normal kidney samples $(n=10)$. B.i - Targeted therapy responders $(n=27)$. B.ii - Targeted therapy resistant $(n=9)$. C.i. - Immunotherapy responders $(n=18)$. C.ii - Immunotherapy resistant $(n=25)$. 


\section{DISCUSSION}

Our geospatial analysis of CAF distribution demonstrated that close-proximity clustering of CAFs and tumor cells potentiates tumor cell proliferation. It also shows that a higher density and staining intensity of CAFs and proliferating tumor cells is associated with poor OS and systemic treatment outcomes. To our knowledge, this is the first ex vivo analysis associating CAFs with the potentiation of tumor cell proliferation in metastatic ccRCC.

We found that CAFs were significantly more clustered with proliferating than apoptotic tumor cells. Overall, these findings, as illustrated in Figure 3, show that close tumor cell proximity to CAFs potentiates proliferation. Additionally, this spatial architecture suggests that tumor cells may be in competition with one another for resources provided by CAFs, with the further tumor cells succumbing to apoptosis; however, this analysis was not designed to directly test this hypothesis.

Though this is the first study to directly measure ex vivo clustering of CAFs with proliferating tumor cells in ccRCC, prior studies have demonstrated this effect in vitro, and several ex vivo analyses have discovered pathways that presumably rely on close proximity [1$3,7,18]$. Notably, CAFs have been found to undergo a metabolic shift when exposed to adjacent cancer cells. This shift results in a Warburg-like glycolytic metabolism in CAFs supplying lactate for cancer cells to fuel the Krebs cycle, which leads to anabolic growth and tumor cell proliferation[7]. Presumably this is a paracrine effect, with CAF-adjacent tumor cells competing with one another for lactate. This metabolic shift is impacted via the HIF1 pathway, which is of particular relevance in ccRCC, a disease in which the vast majority of tumors harbor a somatic alteration in the $\mathrm{VHL}$ gene. The $\mathrm{VHL}$ alteration results in dramatically increased intracellular HIFa, which would be expected to further enhance the aforementioned metabolic shift [26]. This well-described effect of CAFs on the tumor microenvironment lends significant biologic plausibility to the geospatial findings identified in this analysis.

Similarly, hypoxia in the tumor microenvironment has been identified as a significant determinant of the extracellular matrix composition in tumors, resulting in increased HIF1A expression, subsequently increasing the production of growth factors that can potentiate tumor cell proliferation and trigger fibroblast activation and fibrosis [27]. In the $>80 \%$ of ccRCC tumors that harbor somatic $\mathrm{VHL}$ mutations, the resulting derangement of HIFa metabolism would be expected to result in a tumor microenvironment consistent with extreme hypoxic conditions, regardless of the true state of oxygen availability within the tissue[26]. This is another example of significant overlap existing between ccRCC and CAF-potentiated molecular pathways.

Additionally, this analysis of a metastatic cCRCC cohort demonstrated strong associations between increased CAF staining intensity and inferior OS, while accounting for age and IMDC risk category. This finding corroborates previous IHC studies of CCRCC patients that identified an association between increasing CAF density and OS, as well as more advanced stage at diagnosis; however, these cohorts did not include stage IV patients [4-6].

Using Ki-67 staining, this analysis also identified worse OS associated with increased density and staining intensity of proliferating tumor cells. Though not yet adopted into clinical practice, increased Ki-67 staining has been shown in several studies to be associated with worse survival in ccRCC patients across a wide range of clinical stages [14-16,28]. Cell cycle proliferation (CCP) scoring, an RNA-seq-based gene signature score quantifying the expression level of several genes associated with tumor cell proliferation, has been recently described as a promising prognostic biomarker for predicting poor survival and adverse pathology in patients with clinically localized ccRCC [29,30]. Ki-67 IHC staining is a simpler and less costly method 
than the CCP score and may yield similar biologic information. Further studies are warranted to determine agreement and concordance between Ki-67 IHC staining and CCP score.

In addition to survival metrics, we identified several promising associations between geospatial distributions and treatment response and resistance. Patients who were resistant to TT had increased Ki-67 density and H-scores, which to our knowledge has not been previously reported. Additionally, two-dimensional kernel-density plots depicting the NN distances of CAFs to their nearest $\mathrm{Ki}-67^{+}$and caspase- $3^{+}$cellular neighbors yielded density distributions that are clearly disparate between TT- and IT-responding and resistant tumors. The difference between these distributions was primarily driven by the distance from CAFs to their nearest caspase- $3^{+}$ neighbor, with TT responders having significantly longer CAF-caspase NN distance than patients with TT resistance and IT responders having significantly shorter CAF-caspase NN distance than patients with IT resistance. These novel findings suggest the possibility of distinct geospatial CAF architectures associated with response to TT versus IT.

There are several limitations of our study that deserve mention. First, the patient cohort studied was heterogenous and included a variety of systemic treatment agents and was determined partially on the availability of tumor samples, limiting the applicability of our results with contemporary systematic treatment regimens. Second, we used a limited number of cellular markers, which likely oversimplified the underlying biology of the tumor microenvironment. There are limits to the practicality of examining an exhaustive list of cellular markers in a novel study such as this and interpreting their possible clinical impact. However, this simplification did allow us to examine these specific markers in a robust fashion and identify possible spatial interactions deserving of future investigations. Lastly, we limited our study to using 2 of the more commonly employed spatial metrics, Ripley's K and NN-distance, while acknowledging that many other spatial metrics have been defined in the literature.

\section{CONCLUSION}

This ex vivo geospatial analysis of CAF distribution suggests that close-proximity clustering of tumor cells and CAFs potentiates tumor cell proliferation, resulting in worse OS and resistance to TT in metastatic cCRCC. Patients with high aSMA and $\mathrm{Ki}-67 \mathrm{H}$-scores had significantly worse OS from the time of immunotherapy initiation.

\section{ACKNOWLEDGEMENTS}

This study was supported by the Molecular Genomics, Tissue Core, and Biostatistics and Bioinformatics Shared Resources at the H. Lee Moffitt Cancer Center and Research Institute, an $\mathrm{NCl}$ designated Comprehensive Cancer Center (P30-CA076292). Editorial assistance was provided by the Moffitt Cancer Center's Office of Scientific Publishing by Dr. Paul Fletcher \& Daley Drucker. No compensation was given beyond their regular salaries.

\section{DATA AVAILABILITY STATEMENT}

The data that support the findings of this study are available from the corresponding author upon reasonable request. 


\section{CONFLICT OF INTEREST DISCLOSURE}

The corresponding author certifies that all conflicts of interest, including specific financial interests and relationships and affiliations relevant to the subject matter or materials discussed in the manuscript (ie. employment/affiliation, grants or funding, consultancies, honoraria, stock ownership or options, expert testimony, royalties, or patents filed, received, or pending), are the following: GJK, YK, JJ, NC, AH, AC, AMA, LZ, EK, JC, MCF, NF, MF, JD, JYP, LW, and PMA have no disclosures; BJM is an NCCN Kidney Cancer Panel Member; PES is an NCCN Bladder and Penile Cancer Panel Member and Vice-Chair; JM is an Associate Center Director at Moffitt Cancer Center, has ownership interest in Fulgent Genetics, Inc., Aleta Biotherapeutics, Inc., Cold Genesys, Inc., Myst Pharma, Inc., and Tailored Therapeutics, Inc., and is a consultant/advisory board member for ONCoPEP, Inc., Cold Genesys, Inc., Morphogenesis, Inc., Mersana Therapeutics, Inc., GammaDelta Therapeutics, Ltd., Myst Pharma, Inc., Tailored Therapeutics, Inc., Verseau Therapeutics, Inc., Iovance Biotherapeutics, Inc., Vault Pharma, Inc., Noble Life Sciences Partners, Fulgent Genetics, Inc., UbiVac, LLC, Vycellix, Inc., and Aleta Biotherapeutics, Inc.

\section{REFERENCES}

1. Kalluri, R. The biology and function of fibroblasts in cancer. Nat Rev Cancer 2016, 16, 582-598, doi:10.1038/nrc.2016.73.

2. Tao, L.; Huang, G.; Song, H.; Chen, Y.; Chen, L. Cancer associated fibroblasts: An essential role in the tumor microenvironment. Oncol Lett 2017, 14, 2611-2620, doi:10.3892/ol.2017.6497.

3. Harper, J.; Sainson, R.C. Regulation of the anti-tumour immune response by cancer-associated fibroblasts. Semin Cancer Biol 2014, 25, 69-77, doi:10.1016/j.semcancer.2013.12.005.

4. Errarte, P.; Larrinaga, G.; Lopez, J.I. The role of cancer-associated fibroblasts in renal cell carcinoma. An example of tumor modulation through tumor/non-tumor cell interactions. J Adv Res 2020, 21, 103-108, doi:10.1016/j.jare.2019.09.004.

5. Bakhtyar, N.; Wong, N.; Kapoor, A.; Cutz, J.C.; Hill, B.; Ghert, M.; Tang, D. Clear cell renal cell carcinoma induces fibroblast-mediated production of stromal periostin. Eur J Cancer 2013, 49, 3537-3546, doi:10.1016/j.ejca.2013.06.032.

6. $\quad$ Lopez, J.I.; Errarte, P.; Erramuzpe, A.; Guarch, R.; Cortes, J.M.; Angulo, J.C.; Pulido, R.; Irazusta, J.; Llarena, R.; Larrinaga, G. Fibroblast activation protein predicts prognosis in clear cell renal cell carcinoma. Hum Pathol 2016, 54, 100-105, doi:10.1016/j.humpath.2016.03.009.

7. Fiaschi, T.; Marini, A.; Giannoni, E.; Taddei, M.L.; Gandellini, P.; De Donatis, A.; Lanciotti, M.; Serni, S.; Cirri, P.; Chiarugi, P. Reciprocal metabolic reprogramming through lactate shuttle coordinately influences tumor-stroma interplay. Cancer Res 2012, 72, 5130-5140, doi:10.1158/0008-5472.CAN-12-1949.

8. Rini, B.I.; Small, E.J. Biology and clinical development of vascular endothelial growth factortargeted therapy in renal cell carcinoma. J Clin Oncol 2005, 23, 1028-1043, doi:10.1200/JCO.2005.01.186.

9. Scholzen, T.; Gerdes, J. The Ki-67 protein: from the known and the unknown. J Cell Physiol 2000, 182, 311-322, doi:10.1002/(SICI)1097-4652(200003)182:3<311::AID-JCP1>3.0.CO;2-9.

10. Li, L.T.; Jiang, G.; Chen, Q.; Zheng, J.N. Ki67 is a promising molecular target in the diagnosis of cancer (review). Mol Med Rep 2015, 11, 1566-1572, doi:10.3892/mmr.2014.2914.

11. Nielsen, T.O.; Leung, S.C.Y.; Rimm, D.L.; Dodson, A.; Acs, B.; Badve, S.; Denkert, C.; Ellis, M.J.; Fineberg, S.; Flowers, M.; et al. Assessment of Ki67 in Breast Cancer: Updated Recommendations from the International Ki67 in Breast Cancer Working Group. J Natl Cancer Inst 2020, doi:10.1093/jnci/djaa201. 
12. Nishishita, R.; Morohashi, S.; Seino, H.; Wu, Y.; Yoshizawa, T.; Haga, T.; Saito, K.; Hakamada, K.; Fukuda, S.; Kijima, H. Expression of cancer-associated fibroblast markers in advanced colorectal cancer. Oncol Lett 2018, 15, 6195-6202, doi:10.3892/ol.2018.8097.

13. Soliman, N.A.; Yussif, S.M. Ki-67 as a prognostic marker according to breast cancer molecular subtype. Cancer Biol Med 2016, 13, 496-504, doi:10.20892/j.issn.2095-3941.2016.0066.

14. Xie, Y.; Chen, L.; Ma, X.; Li, H.; Gu, L.; Gao, Y.; Fan, Y.; Zhang, Y.; Zhang, X. Prognostic and clinicopathological role of high $\mathrm{Ki}-67$ expression in patients with renal cell carcinoma: a systematic review and meta-analysis. Sci Rep 2017, 7, 44281, doi:10.1038/srep44281.

15. Gayed, B.A.; Youssef, R.F.; Bagrodia, A.; Darwish, O.M.; Kapur, P.; Sagalowsky, A.; Lotan, Y.; Margulis, V. Ki67 is an independent predictor of oncological outcomes in patients with localized clear-cell renal cell carcinoma. BJU Int 2014, 113, 668-673, doi:10.1111/bju.12263.

16. Bui, M.H.; Visapaa, H.; Seligson, D.; Kim, H.; Han, K.R.; Huang, Y.; Horvath, S.; Stanbridge, E.J.; Palotie, A.; Figlin, R.A.; et al. Prognostic value of carbonic anhydrase IX and $\mathrm{KI} 167$ as predictors of survival for renal clear cell carcinoma. J Urol 2004, 171, 2461-2466, doi:10.1097/01.ju.0000116444.08690.e2.

17. Motzer, R.J.; Jonasch, E.; Michaelson, M.D.; Nandagopal, L.; Gore, J.L.; George, S.; Alva, A.; Haas, N.; Harrison, M.R.; Plimack, E.R.; et al. NCCN Guidelines Insights: Kidney Cancer, Version 2.2020. J Natl Compr Canc Netw 2019, 17, 1278-1285, doi:10.6004/jnccn.2019.0054.

18. Chen, X.; Song, E. Turning foes to friends: targeting cancer-associated fibroblasts. Nat Rev Drug Discov 2019, 18, 99-115, doi:10.1038/s41573-018-0004-1.

19. Rizzardi, A.E.; Johnson, A.T.; Vogel, R.I.; Pambuccian, S.E.; Henriksen, J.; Skubitz, A.P.; Metzger, G.J.; Schmechel, S.C. Quantitative comparison of immunohistochemical staining measured by digital image analysis versus pathologist visual scoring. Diagn Pathol 2012, 7, 42, doi:10.1186/1746-1596-7-42.

20. McClelland, R.A.; Finlay, P.; Walker, K.J.; Nicholson, D.; Robertson, J.F.; Blamey, R.W.; Nicholson, R.I. Automated quantitation of immunocytochemically localized estrogen receptors in human breast cancer. Cancer Res 1990, 50, 3545-3550.

21. Marcon, E.; Puech, F. A typology of distance-based measures of spatial concentration. Regional Science and Urban Economics 2017, 62, 56-67, doi:https://doi.org/10.1016/i.regsciurbeco.2016.10.004.

22. Chakiryan, N.H.; Kimmel, G.J.; Kim, Y.; Hajiran, A.; Aydin, A.M.; Zemp, L.; Katende, E.; Nguyen, J.; Lopez-Blanco, N.; Chahoud, J.; et al. Spatial clustering of CD68+ tumor associated macrophages with tumor cells is associated with worse overall survival in metastatic clear cell renal cell carcinoma. PLoS One 2021, 16, e0245415, doi:10.1371/journal.pone.0245415.

23. Warren, A.Y.; Harrison, D. WHO/ISUP classification, grading and pathological staging of renal cell carcinoma: standards and controversies. World J Urol 2018, 36, 1913-1926, doi:10.1007/s00345018-2447-8.

24. Heng, D.Y.; Xie, W.; Regan, M.M.; Harshman, L.C.; Bjarnason, G.A.; Vaishampayan, U.N.; Mackenzie, M.; Wood, L.; Donskov, F.; Tan, M.H.; et al. External validation and comparison with other models of the International Metastatic Renal-Cell Carcinoma Database Consortium prognostic model: a population-based study. Lancet Oncol 2013, 14, 141-148, doi:10.1016/S1470-2045(12)70559-4.

25. Faraggi, D.; Simon, R. A simulation study of cross-validation for selecting an optimal cutpoint in univariate survival analysis. Stat Med 1996, 15, 2203-2213, doi:Doi 10.1002/(Sici)10970258(19961030)15:20<2203::Aid-Sim357>3.3.Co;2-7.

26. Gnarra, J.R.; Tory, K.; Weng, Y.; Schmidt, L.; Wei, M.H.; Li, H.; Latif, F.; Liu, S.; Chen, F.; Duh, F.M.; et al. Mutations of the VHL tumour suppressor gene in renal carcinoma. Nat Genet 1994, 7, 8590, doi:10.1038/ng0594-85. 
27. Gilkes, D.M.; Semenza, G.L.; Wirtz, D. Hypoxia and the extracellular matrix: drivers of tumour metastasis. Nat Rev Cancer 2014, 14, 430-439, doi:10.1038/nrc3726.

28. Mehdi, M.Z.; Nagi, A.H.; Naseem, N. MCM - 2 and Ki - 67 as proliferation markers in renal cell carcinoma: A quantitative and semi - quantitative analysis. Int Braz J Urol 2016, 42, 1121-1128, doi:10.1590/S1677-5538.IBJU.2015.0388.

29. Morgan, T.M.; Mehra, R.; Tiemeny, P.; Wolf, J.S.; Wu, S.; Sangale, Z.; Brawer, M.; Stone, S.; Wu, C.L.; Feldman, A.S. A Multigene Signature Based on Cell Cycle Proliferation Improves Prediction of Mortality Within 5 Yr of Radical Nephrectomy for Renal Cell Carcinoma. Eur Urol 2018, 73, 763-769, doi:10.1016/j.eururo.2017.12.002.

30. Tosoian, J.J.; Feldman, A.S.; Abbott, M.R.; Mehra, R.; Tiemeny, P.; Wolf, J.S., Jr.; Stone, S.; Wu, S.; Daignault-Newton, S.; Taylor, J.M.G.; et al. Biopsy Cell Cycle Proliferation Score Predicts Adverse Surgical Pathology in Localized Renal Cell Carcinoma. Eur Urol 2020, 78, 657-660, doi:10.1016/j.eururo.2020.08.032. 